Reprod. Nutr. Dévelop., 1986, 26 (5 B), 1163-1176.

\title{
Les lipases du tractus digestif
}

\author{
Y. GARGOURI ('), G. PIÉRONI ('), H. MOREAU, Francine FERRATO (') \\ C. RIVIĖRE ( $\left.{ }^{1}\right)$, J.-F. SAUNIĖRE $\left({ }^{2}\right)$, P. A. LOWE $\left({ }^{3}\right)$, L. SARDA $\left({ }^{4}\right)$, \\ R. VERGER ( $\left.{ }^{1}\right)$
}

(1) Centre de Biochimie et de Biologie Moléculaire du CNRS, 31, Chemin Joseph-Aiguier, 13402 Marseille Cedex 9, France.

(2) Laboratoire d'Hépatogastrœentérologie, Hôpital Sainte-Marguerite, 270, boulevard de Sainte-Marguerite, 13009 Marseille, France.

(3) Celltech Ltd, 230-250 Bath Road, Slough, SL1 4DY, Berkshire, UK.

(4) Laboratoire de Biochimie, Faculté des Sciences Saint-Charles, place Victor-Hugo, 13003 Marseille, France.

\section{Summary. Lipases of gastrointestinal tract.}

Studies on gastrointestinal lipolysis have underestimated several important points. In view of recent in vitro data obtained in our laboratories, this review focuses on the role of gastric lipolysis during fat digestion. Polyclonal antibodies generated from purified rat lingual lipase were used to screen a cDNA library prepared from mRNA isolated from the serous glands of rat tongue cloned in $E$. coli expression vectors. A cDNA clone was isolated and the nucleotide and predicted amino acid sequences obtained. Comparison with the N-terminal amino acid sequence of the purified enzyme confirmed the identity of the cDNA. The amino acid sequence of rat lingual lipase consisted of 377 residues and showed little homology with porcine pancreatic lipase, apart from a short region containing a serine residue at an analogous position to the Ser 152 of the porcine enzyme. Human gastric lipase activity on tributyrin emulsion was detected only in the presence of amphiphiles. This behaviour was in sharp contrast with the strong inhibitory effect of amphiphiles observed on pure pancreatic lipase. To reveal human gastric lipase activity, amphiphiles must be added to human gastric lipase in order to prevent irreversible interfacial denaturation. Human gastric lipase activity was found to be restricted to triacylglycerol/water surface tensions ranging from 8 to 13 dynes/cm. All amphiphiles which decrease interfacial tension to less than 8 dynes $/ \mathrm{cm}$ act as irreversible inhibitors of human gastric lipase in the absence or presence of bile salts. Our results confirm that human gastric lipase is capable of hydrolysing triacylglycerol in the presence of the bile salts concentration prevailing in the upper small intestine and in the presence of alimentary proteins. These observations could explain the high dietary lipid absorption observed under pancreatic lipase deficiency.

\section{Introduction.}

Les triglycérides à longues chaînes représentent la forme principale des lipides de l'alimentation humaine et des animaux supérieurs pour lesquels ils constituent une source importante d'énergie chimique.

Reproduction, Nutrition, Développement, $n^{\circ} 5$ B-86. -2 
La digestion des triglycérides alimentaires, insolubles dans l'eau, comprend trois étapes essentielles : a) la dispersion sous forme de particules émulsifiées ; b) I'hydrolyse enzymatique par les lipases du tractus gastrointestinal à l'interface lipide-eau ; c) la désorption des produits de la réaction de lipolyse, monoglycérides et acides gras et leur dispersion dans la phase aqueuse du contenu intestinal, sous forme de solution micellaire mixte (Hofmann et Borgström, 1962) ou sous forme d'une phase liquide-cristalline (Patton et Carey, 1979; Lindström et al., 1981 ; Patton, 1981 ; Carey et al., 1983), avec les sels biliaires de la bile.

Les connaissances actuelles concernant les aspects biochimiques fondamentaux de la digestion des lipides alimentaires ont été acquises en grande partie grâce aux travaux réalisés sur la lipase pancréatique. Cependant, les études effectuées jusqu'ici sur la digestion gastrointestinale des triglycérides, n'ont pas abordé - ou ont largement sous-estimé - plusieurs points importants. Tout d'abord, il est évident que les forces mécaniques responsables in vivo de l'émulsification des lipides alimentaires sont faibles. La proportion des triglycérides alimentaires hydrolysés et absorbés in vivo est plus grande après l'ingestion d'un repas préémulsifié, qu'après l'ingestion d'une même quantité de graisses alimentaires sous forme non émulsifiée. Un autre point, largement sous-estimé, est la présence dans le tractus digestif de composés amphipathiques autres que les sels biliaires (phospholipides, protéines). II est bien connu que les sels biliaires purs sont de très mauvais émulsifiants des triglycérides purs. L'existence d'une lipase linguale suggère que l'hydrolyse des triglycérides débuterait dans l'estomac. Cette réaction serait la première étape de la digestion des lipides alimentaires; les produits amphiphiles résultant de cette hydrolyse faciliteraient l'émulsification des triglycérides (Hamosh et al. 1975). Cette hypothèse a été directement confirmée par les expériences de Linthorst et al. (1977) qui ont clairement montré que le mélange de sels biliaires et des produits de la lipolyse, présents dans l'intestin, participait à l'émulsification des triglycérides dans des conditions de faibles contraintes mécaniques. Une autre conséquence importante de la préhydrolyse des lipides alimentaires par les lipases préduodénales est que les diglycérides formés constituent, avec les triglycérides non hydrolysés, l'un des substrats physiologiques importants de la lipase pancréatique.

Chez l'homme, les canaux biliaire et pancréatique confluent dans le duodenum au niveau de l'ampoule de Vater. Serling (1954) a noté que $58 \%$ des humains possédaient une jonction commune de ces deux canaux de sécrétion. De plus, il est bien établi que les sécrétions biliaire et pancréatique sont simultanément déclenchées par le même stimulus hormonal (Jorpes et Mutt, 1966). Ces données anatomiques et physiologiques plaident en faveur d'une association entre lipase, colipase et constituants biliaires avant l'étape de lipolyse, c'est-à-dire en l'absence de tout substrat hydrolysable (Lairon et al., 1978), alors que les expériences habituellement réalisées in vitro le sont selon une chronologie inverse : triglycérides, sels biliaires, et finalement lipase-colipase. Les conséquences éventuelles de cet ordre d'addition méritent d'être prises en compte lors de l'interprétation de la plupart des données de la littérature. 


\section{Clonage et détermination de la séquence nucléotidique du cDNA de la lipase linguale de rat}

Chez le rat, les glandes séreuses (glandes de von Ebner) localisées dans la partie postérieure de la langue sécrètent une lipase acide qui hydrolyse les triglycérides en diglycérides, monoglycérides et acides gras libres dans une zone de $\mathrm{pH}$ comprise entre 2 et 6,5 (Hamosh, 1984). Les produits de la lipolyse facilitent l'émulsification et I'hydrolyse ultérieure des lipides alimentaires. Des activités lipasiques stables en milieu acide ont été détectées dans des homogénats de glandes de von Ebner et dans des aspirats gastriques humains (Hamosh et Scow, 1973 ; Hamosh et Burns, 1977 ; Tiruppathi et Balasubramanian, 1982 ; Carey et al., 1983). La lipase linguale de rat possède une masse moléculaire d'environ 50000 daltons (Field et Scow, 1983 ; Roberts et al., 1984), valeur très voisine de celle de la lipase pancréatique de porc (De Caro et al., 1981).

Chez l'homme, dans diverses conditions physiologiques ou pathologiques, telles que les fibroses cystiques, les pancréatites, la naissance prématurée ou l'alcoolisme, des insuffisances en lipase pancréatique ont été décrites. Une enzymothérapie de substitution utilisant des lipases acides a été préconisée (Abrams et al., 1984). L'expression dans des microorganismes d'un cDNA cloné codant pour la lipase gastrique humaine pourrait être une source de lipase, résistante en milieu acide, utilisable par voie orale en thérapeutique humaine.

Différentes techniques de clonage ont été utilisées pour déduire la séquence des aminoacides d'un très grand nombre de protéines. Très souvent, les clones ont été isolés à partir d'une banque de cDNA grâce à l'utilisation de sondes oligonucléotidiques dont la synthèse a été réalisée à partir des données provenant de la séquence protéique (Suggs et al., 1981). Une stratégie alternative, très utile en l'absence de toute séquence protéique, consiste à construire une banque de cDNA dans des vecteurs qui autorisent la transcription partielle en mRNA qui sera ultérieurement traduit sous forme protéique, généralement après fusion avec une protéine bactérienne.

Les premiers essais ont été conduits avec des clones de $E$. coli synthétisant la lipase linguale de rat qui ont été identifiés grâce à des anticorps polyclonaux réagissant avec la protéine dénaturée et non glycosylée (Docherty et al., 1985). Le cDNA préparé à partir d'un mRNA codant pour une protéine de 40000 daltons reconnue par ces anticorps a été divisé en deux lots. L'un de ceux-ci a été digéré par Sau3A. Sur l'autre, ont été fixés des " bouts collants" BamHI. La région codant pour la lipase linguale de rat contient 3 fragments Sau3A, chacun d'entre eux débutant par un cadre de lecture différent. Tous les trois ont été fusionnés correctement avec le vecteur approprié. De plus, l'efficacité de détection par les anticorps est similaire pour les deux lots de CDNA. Ceci indique que les vecteurs utilisés, les antisera et les procédés de détection sont suffisamment efficaces pour permettre une bonne détection des clones de cDNA codant pour la lipase linguale de rat. L'étape de lyse par l'urée est probablement utile pour la dissolution des produits de fusion avec la $\beta$-galactosidase, les rendant plus accessibles à la détection par les anticorps. Les cellules d'E. coli transformées biosynthétisent de 5 à 
$10 \%$ de leur masse protéique totale sous la forme d'une protéine de fusion, identifiée par Western-blotting (Docherty et al., 1985).

Le cDNA identifié grâce aux anticorps anti-lipase linguale de rat correspond effectivement au mRNA de lipase linguale de rat, comme le montre la comparaison de sa séquence nucléotidique avec la séquence $\mathrm{N}$-terminale de la protéine purifiée à partir de la langue de rat. La séquence d'aminoacides prédite à partir de la séquence nucléotidique du cDNA cloné indique que l'enzyme mature est composé de 377 résidus aminoacides. Le poids moléculaire calculé à partir de cette séquence est de 42564 daltons ; cette valeur est en bon accord avec celles déterminées par électrophorèse en présence de sodium dodécylsulfate pour les protéines, native et déglycosylée, ou synthétisée in vitro. La lipase linguale contient 5 sites potentiels de $\mathrm{N}$-glycosylation (Asn-X-Ser ou Thr). A en juger par le profil électrophorétique de l'enzyme déglycosylé à l'aide de l'EndoH, il semble très probable qu'au moins trois de ces sites soient effectivement glycosylés dans l'enzyme natif.

La séquence de la lipase linguale de rat a été comparée à celle de la lipase pancréatique de porc. L'enzyme d'origine linguale possède soixante-douze résidus de moins que l'enzyme pancréatique et l'homologie entre les séquences des deux protéines est très faible. II existe seulement une certaine similitude entre les deux enzymes au niveau de la sérine 152. Cette sérine, dans la lipase pancréatique, réagit avec le diéthyl-para-nitrophényl phosphate sous forme micellaire et pourrait être impliquée dans la fixation de l'enzyme aux interfaces (Chapus et Sémériva, 1976). Ce résidu sérine est présent dans la séquence Gly-His-Ser-Leu-Gly de la lipase pancréatique de porc et en position équivalente dans la lipase linguale de rat: Gly-His-Ser-Gln-Gly. Le seul point commun entre les deux enzymes se situe au niveau du seul site de glycosylation de la lipase pancréatique de porc (Asn-GlyThr) et d'un site potentiel correspondant dans l'enzyme linguale : (Asn-Pro-Thr).

Dans l'avenir, le cDNA de la lipase linguale de rat devrait permettre d'isoler des clones spécifiques de l'enzyme humain homologue.

\section{La lipase gastrique humaine : influence des composés amphiphiles}

L'hydrolyse des triglycérides alimentaires commence dans l'estomac et est catalysée par des lipases stables en milieu acide présentes dans le suc gastrique (Schonheyder et Volquartz, 1946 ; Siurala, 1956 ; Borgström et al., 1957 ; Popiela et al., 1965 ; Eastwood et Hamilton, 1970 ; Cohen et al., 1971 ; Hamosh et al., 1978 ; Muller et al., 1975 ; Jensen et al., 1982). L'activité lipolytique décelée dans le suc gastrique humain pourrait provenir de différents organes, langue, pharynx ou estomac. Cette question est toujours controversée (Hamosh, 1984). Dès les premiers travaux, qui remontent au début du siècle, l'activité lipase trouvée au niveau de l'estomac fut dénommée lipase gastrique (Volhard, 1901 ; Schonheyder et Volquartz, 1946). Plus tard, I'activité lipasique principale fut attribuée chez I'homme à un enzyme de la sécrétion des glandes de von Ebner, localisées dans la partie postérieure de la langue ; cet enzyme fut dénommé lipase linguale, par analogie avec la lipase linguale de rat (Hamosh et al., 1975 ; Sterling, 1954 ; Jensen et al., 1982 ; Hamosh, 1979 ; Hamosh et al., 1981). Néanmoins, la preuve défini- 
tive de l'existence d'une lipase biosynthétisée par la muqueuse gastrique humaine a été récemment apportée par le clonage, à partir du mRNA extrait de cette muqueuse, d'un cDNA codant pour une protéine dont la séquence est identique à celle des trente premiers résidus de la région $\mathrm{N}$-terminale de la lipase gastrique humaine (Bodmer et al., communication personnelle). Cependant, l'importance physiologique et l'existence elle-même d'une lipase linguale humaine restent encore à établir définitivement.

Une différence majeure entre les comportements des lipases pancréatique et gastrique concerne leurs stabilités et leurs activités respectives aux $\mathrm{pH}$ acides. En effet, il est bien établi que le $\mathrm{pH}$ des aspirats gastriques humains se situe entre 1 et 3 (Hamosh, 1984). La lipase gastrique est particulièrement stable dans de telles conditions. Au contraire, la lipase pancréatique perd de façon irréversible son activité enzymatique aux pH inférieurs à 5 (Verger, 1984). Le pH optimal d'activité

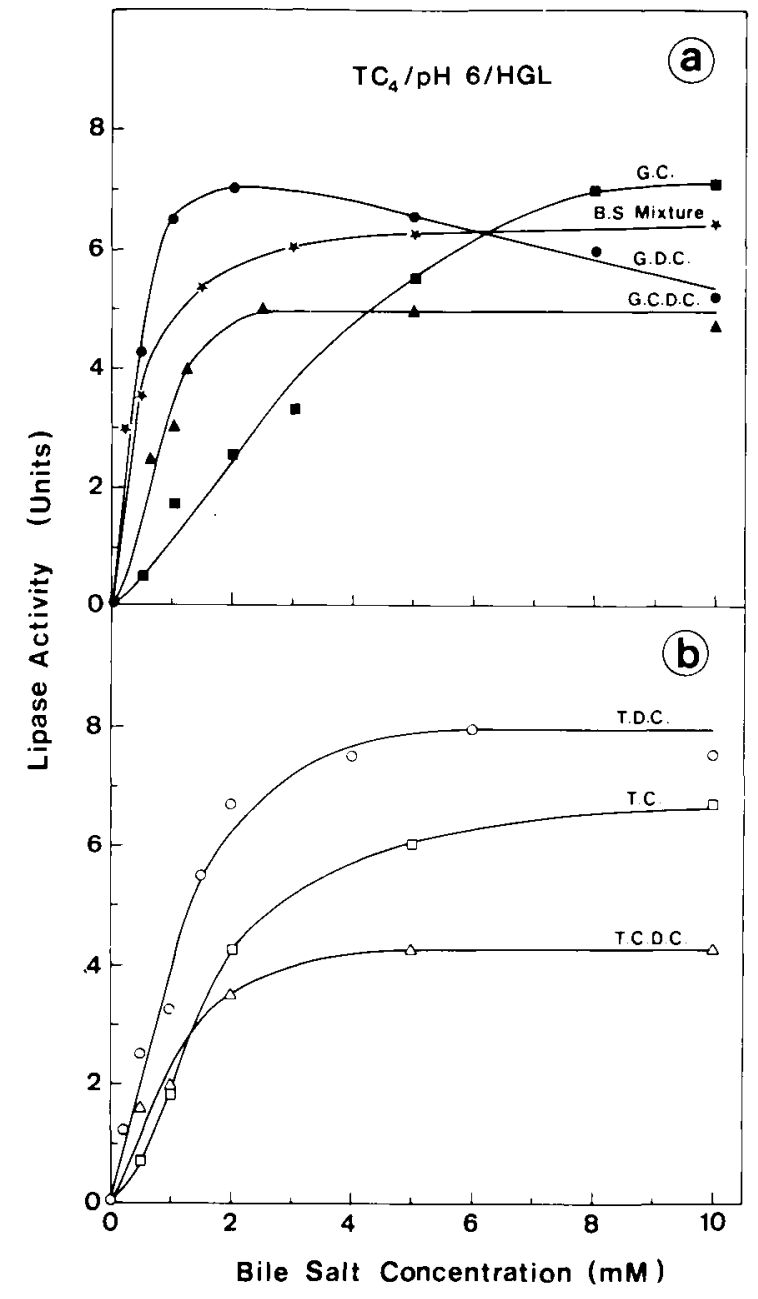

FIG. 1. - Influence de concentrations croissantes de différentes sels biliaires sur la vitesse d'hydrolyse d'une émulsion de tributyrine (TC4) par la lipase gastrique humaine (concentration finale $20 \mathrm{nM})(\mathrm{HGL})$.

- 1a: (1) GC, glycolate: $(\bullet)$ GDC, glycodésoxycholate : (A) GCDC, glycochénodésoxycholate ; (*) mélange de sels biliaires: $\mathrm{GC}$, $29,8 \%$; GCDC, $24,5 \%$; GDC, $11,9 \%$; TC, $12,6 \%$; TCDC, $13,6 \% ;$ TDC, $7,6 \%$

- $1 b:(0)$ TDC, taurodésoxycholate ; (D) TC, taurocholate ; $(\Delta)$ TCDC, taurochénodésoxycholate. 
de la lipase gastrique humaine est de 5,4 tandis que celui de la lipase pancréatique se situe autour de 8-9 (Hamosh, 1984 ; Verger, 1984).

D'autre part, il est établi que les sels biliaires sont de puissants inhibiteurs de l'activité de la lipase pancréatique. Au contraire, ces mêmes sels biliaires semblent agir comme activateurs de la lipase gastrique humaine lorsque l'on utilise de la tributyrine comme substrat (Hamosh, 1984 ; Tiruppathi et Balasubramanian, 1982, fig. 1).

Nous avons étudié l'influence de différents sels biliaires présents dans la bile humaine et de quelques protéines sur l'activité hydrolytique de la lipase gastrique humaine. En l'absence de tout amphiphile, aucune activité enzymatique ne peut être décelée sur une émulsion de tributyrine (fig. 1). Cette absence d'activité enzymatique résulte probablement d'une dénaturation rapide de la lipase gastrique humaine pure, dénaturation résultant de son dépliement à l'interface tributyrine/eau. L'addition ultérieure de divers amphiphiles ne permet pas de restaurer l'activité enzymatique. Cette dénaturation interfaciale irréversible peut être

$\mathrm{TC}_{4} / \mathrm{pH} 6$

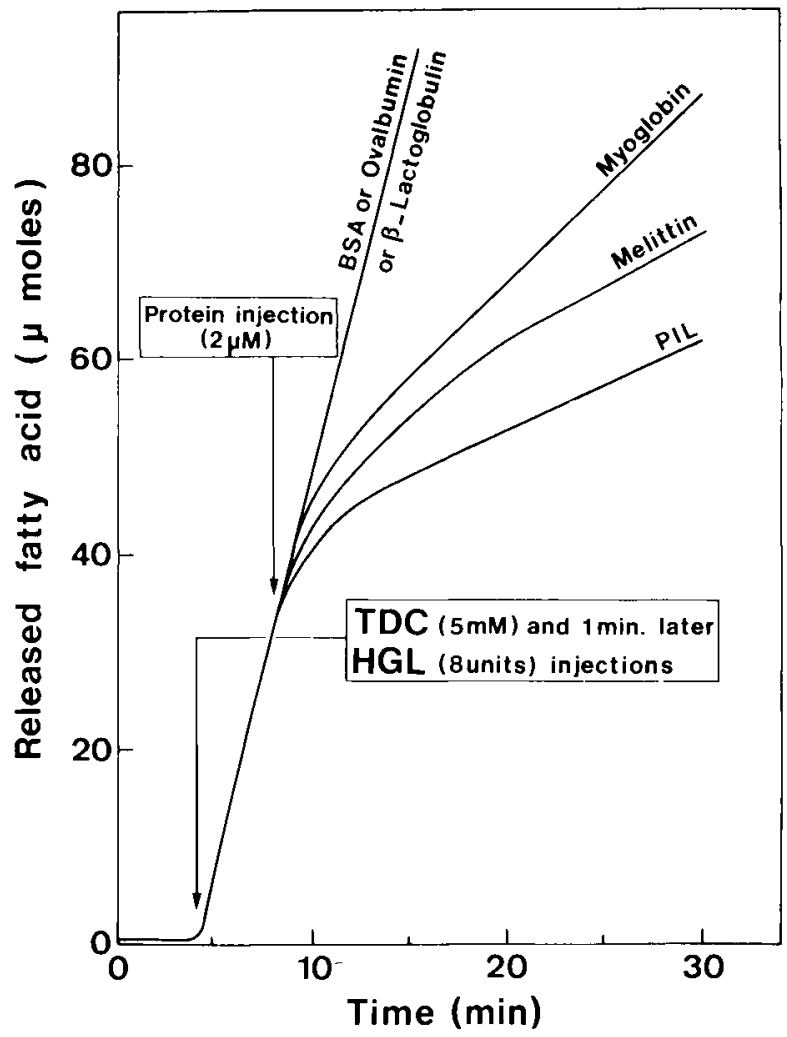

FIG. 2. - Influence de différentes protéines sur la vitesse d'hydrolyse d'une émulsion de tributyrine (TC4).

Une minute après l'addition de TDC (concentration finale $5 \mathrm{mM}$ ), la lipase gastrique humaine (concentration finale $20 \mathrm{nM}$ ) est injectée dans le milieu réactionnel et 4 min plus tard, les protéines sont introduites à la concentration finale de $2 \mu \mathrm{M}$. 
corrélée avec l'énergie interfaciale du système tributyrine/eau ainsi qu'avec la structure probablement flexible de la lipase gastrique humaine. Grâce à la détermination récente de la séquence du gène de l'enzyme (Bodmer et al., communication personnelle), nous savons que la lipase gastrique humaine contient seulement trois résidus de cystéine pour un poids moléculaire de la partie protéique de 43162 daltons. Il est bien connu que les ponts disulfures stabilisent la structure tridimensionnelle des protéines et limitent leur dépliement aux interfaces; d'autre part, il a été établi que les lipases pouvaient se dénaturer plus ou moins rapidement selon les propriétés physico-chimiques interfaciales de leurs substrats. On peut donc comprendre pourquoi la lipase gastrique humaine, réticulée au plus par un pont disulfure, se dénature très rapidement aux interfaces, contrairement à la lipase pancréatique qui possède six ponts disulfure et peut s'adsorber et hydrolyser les émulsions de triglycérides en l'absence de tout additif tel que colipase ou sels biliaires (De Caro et al., 1981, Verger, 1984).

Nous pouvons donc conclure que, pour mesurer l'activité enzymatique de la lipase gastrique humaine sur une émulsion de tributyrine pure, il est nécessaire de prévenir sa dénaturation interfaciale par addition préalable d'amphiphiles, tels que des sels biliaires et/ou des protéines (fig. 2 et 3) ou des phospholipides (fig. 4).

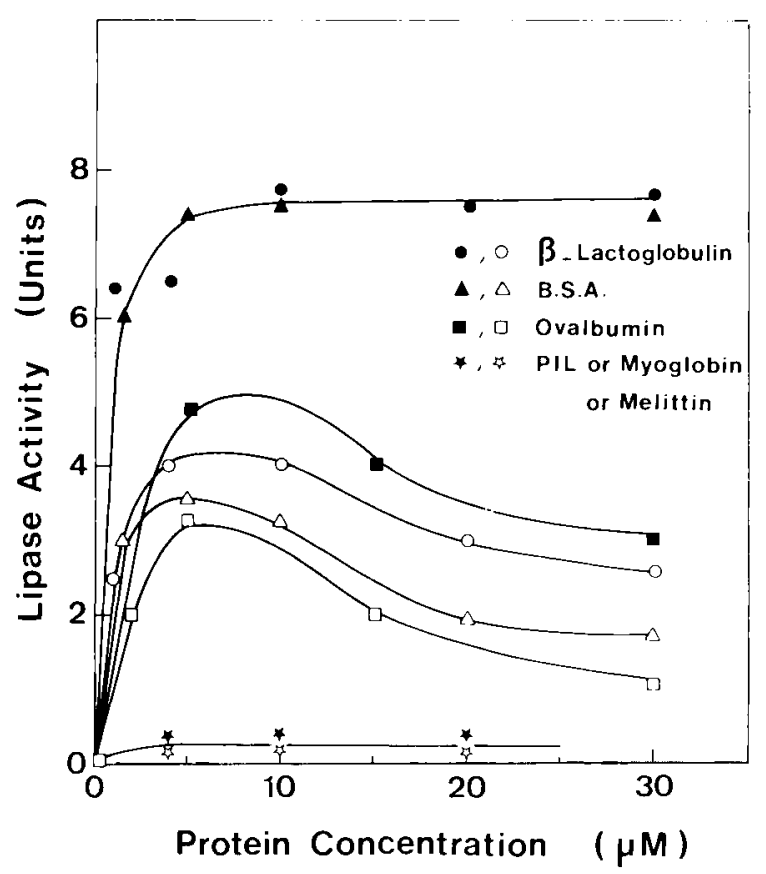

FIG. 3. - Influence de concentrations croissantes de diverses protéines sur la vitesse d'hydrolyse d'une émulsion de tributyrine par la lipase gastrique humaine (concentration finale $20 \mathrm{nM}$ ). Les mesures sont réalisées soit en l'absence (symboles vides), soit en présence (symboles pleins) de $5 \mathrm{mM}$ de TDC.

Les protéines sont ajoutées 2 min avant l'injection de l'enzyme qui est suivie 5 min plus tard par une addition de TDC (concentration finale $5 \mathrm{mM}$ ). L'activité lipase est mesurée à sa valeur maxi-

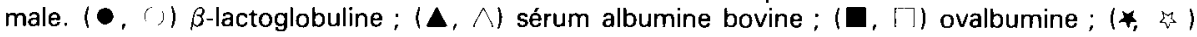
Protéine Inhibitrice de la Lipase (PIL) ou myoglobine ou mélitine. 


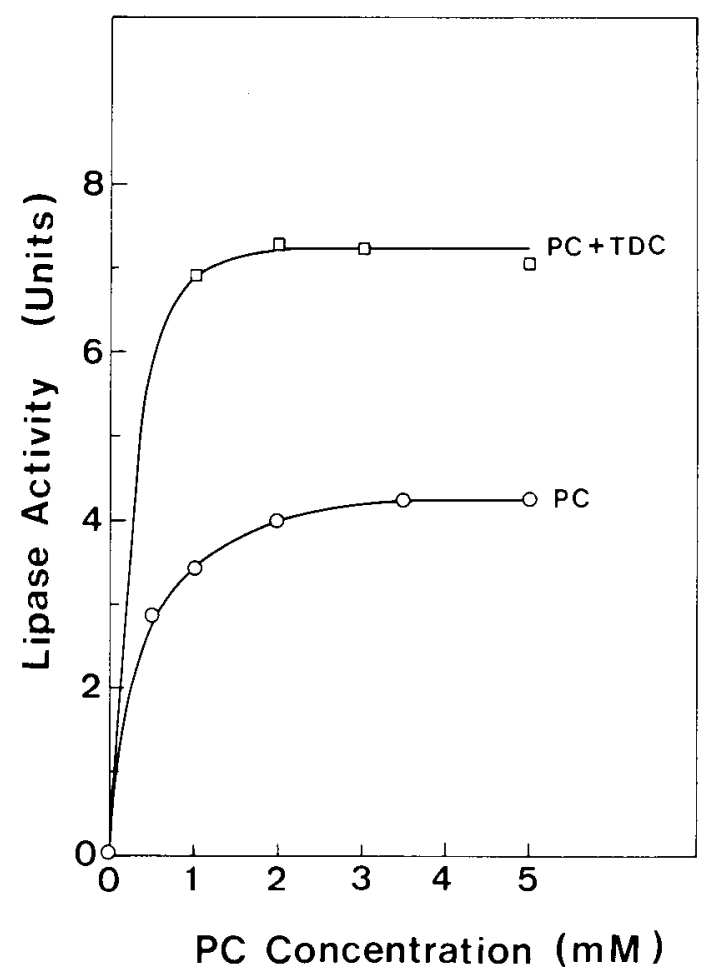

FIG. 4. - Influence de concentrations croissantes de lécithine de jaune d'auf (O) ou de lécithine en présence d'une concentration constante $(5 \mathrm{mM})$ de TDC ( $\square)$ sur la vitesse d'hydrolyse d'une émulsion de tributyrine par la lipase gastrique humaine (concentration finale $20 \mathrm{nM}$ ) additionnée 1 min après les lécithines.

Aucun changement de l'activité lipasique n'est observé lorsque le TDC ou le mélange de sels biliaires (concentration finale $5 \mathrm{mM}$ ) sont ajoutés $5 \mathrm{~min}$ après la lipase gastrique humaine en présence de lécithines.

Pour tenter d'expliquer rationnellement l'influence des différents amphiphiles sur l'activité de la lipase gastrique humaine, nous pouvons utiliser la tension interfaciale comme paramètre de référence. Les résultats obtenus par Gargouri et al., (1986a) et présentés sur la figure 5, montrent clairement que l'activité de la lipase gastrique humaine ne s'exprime que dans une zone de tension interfaciale comprise entre 8 et $13 \mathrm{dynes} / \mathrm{cm}$. La tension à l'interface tributyrine/eau est de 15 dynes $/ \mathrm{cm}$. Cette valeur est donc incompatible avec le maintien d'une structure native et active de la lipase gastrique humaine. Au-dessous de $6 \mathrm{dynes} / \mathrm{cm}$, valeur obtenue en présence de détergents de synthèse ou de protéines particulièrement tensioactives telles que la mélitine ou la myoglobine, aucune activité lipasique n'est décelable. II est intéressant de remarquer que la plupart des protéines alimentaires ainsi que les sels biliaires font décroître la tension interfaciale tributyrine/eau jusqu'à des valeurs comprises entre 8 et 13 dynes $/ \mathrm{cm}$, ce qui représente des conditions permettant l'expression de l'activité enzymatique de la lipase gastrique humaine. 

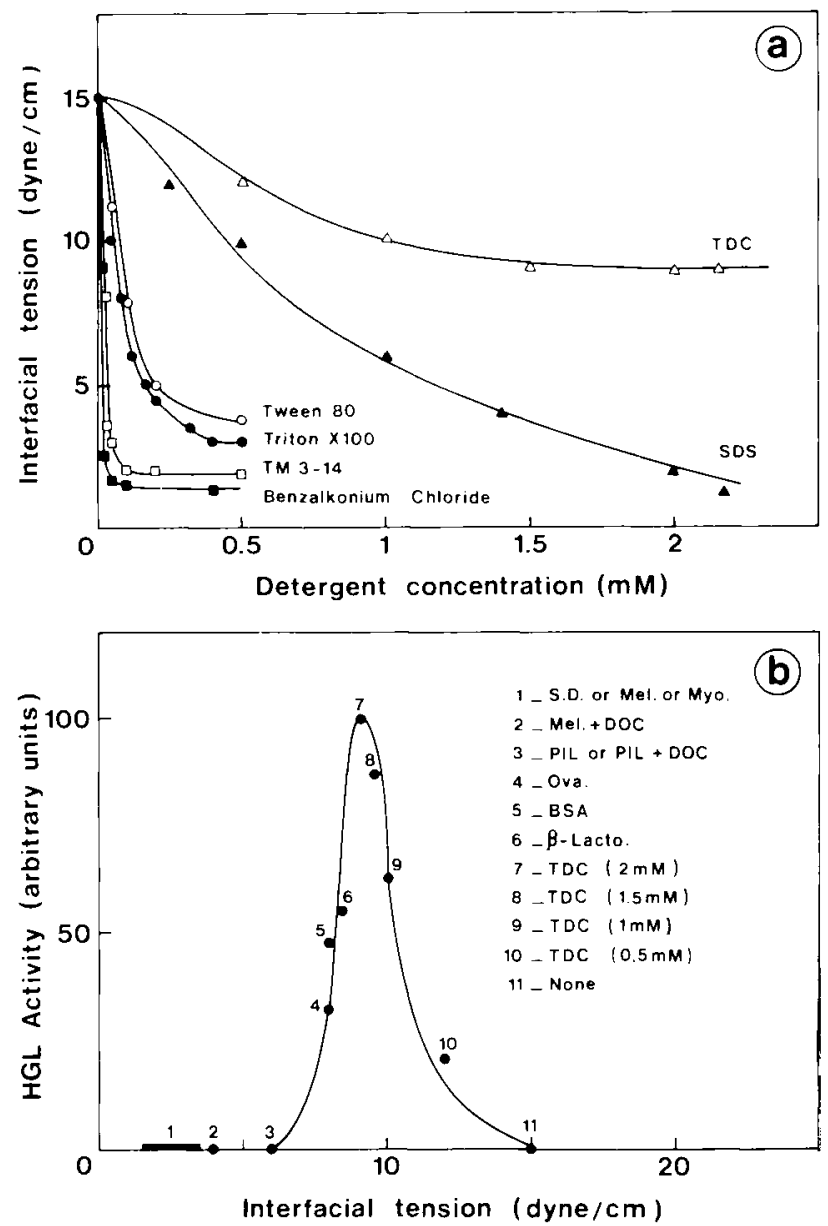

FIG. 5. - Influence de divers amphiphiles sur la tension interfaciale trig/ycéride/eau. Corrélation avec l'activité enzymatique de la lipase gastrique humaine.

- 5a: Influence de concentrations croissantes de différents détergents de synthèse et de TDC sur la tension interfaciale trioléine/eau.

- 5b: Dépendance de l'activité de la lipase gastrique humaine à l'égard de la tension interfaciale en présence de détergents de synthèse ou de TDC ou de protéines (Gargouri et al., 1984, Gargouri et al., 1986a). Cent pour cent de l'activité lipase (unités arbitraires) représentent l'activité enzymatique maximale mesurée en présence de $2 \mathrm{mM}$ de TDC.

Les différences dans la résistance à la dénaturation interfaciale de lipases d'origines diverses peuvent permettre d'expliquer les effets opposés des amphiphiles sur l'activité de l'enzyme gastrique par rapport à l'enzyme pancréatique (Gargouri et al., 1983, 1984a, 1984b, 1986a ; Klein et al., 1967 ; Patton et Carey, 1981).

Nos résultats confirment et précisent, d'une part, des études antérieures montrant que la lipase gastrique humaine est capable d'hydrolyser les triglycérides en présence de concentrations physiologiques de sels biliaires ou de protéines 
alimentaires que I'on trouve habituellement dans la lumière intestinale au cours d'un repas. Ils permettent d'expliquer d'autre part pourquoi, chez l'homme, lors de déficiences en lipase d'origine pancréatique, les triglycérides alimentaires sont absorbés efficacement (Hamosh, 1984, Muller et al., 1975). Nos études, réalisées in vitro indiquent également que certaines protéines alimentaires particulièrement tensioactives du soja (PIL) pourraient avoir une influence négative sur la lipolyse intragastrique in vivo. (Gargouri et al., 1984a, 1986a).

La lipase gastrique humaine purifiée est capable de catalyser à des vitesses comparables l'hydrolyse des triglycérides à chaînes courtes ou longues dans les conditions de $\mathrm{pH}$ que I'on trouve au niveau du contenu gastrique. Nous avons pu mesurer à pH 5,4 des activités spécifiques de $1200 \mu \mathrm{moles} / \mathrm{min} / \mathrm{mg}$ d'enzyme sur tributyrine et de $600 \mu \mathrm{moles} / \mathrm{min} / \mathrm{mg}$ d'enzyme sur huile de soja émulsifiée par des lécithines de jaune d'œuf (Intralipide) (Gargouri et al., 1986b).

Différents auteurs ont remarqué que la lipase pancréatique était totalement inactive sur l'émulsion d'Intralipide utilisée comme substrat (Klein et al., 1967), contrairement à ce qui est observé avec la lipase gastrique humaine (Gargouri et
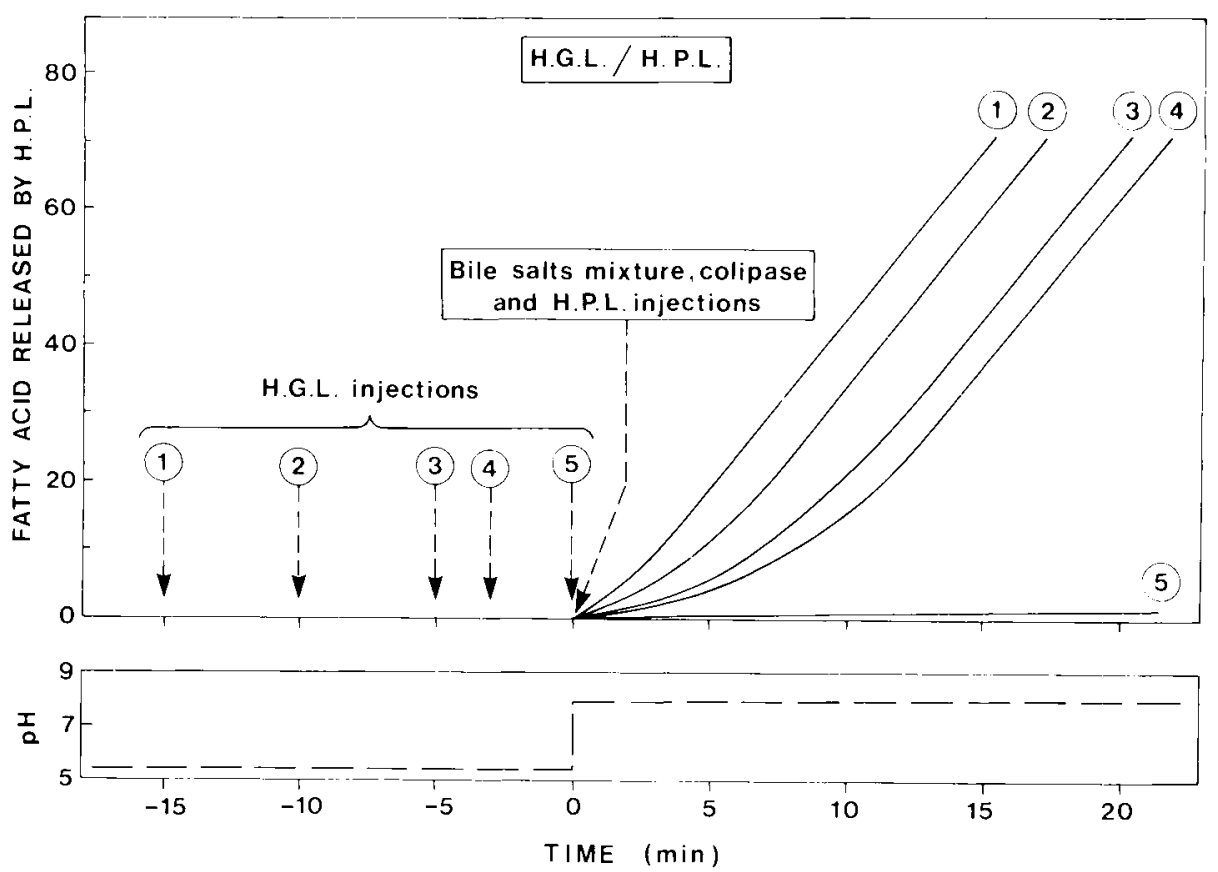

FIG. 6 - Cinétiques d'hydrolyse d'une émulsion d'Intralipide par la lipase pancréatique humaine pure après différentes périodes d'incubation avec de la lipase gastrique humaine pure.

Les flèches représentent les injections de lipase gastrique $(10 \mu \mathrm{g})$ à $\mathrm{pH} 5,4$. Au temps 0 , le $\mathrm{pH}$ est amené de 5,4 à 8,0 et l'on injecte simultanément un mélange de sels biliaires (concentration finale $8 \mathrm{mM})$, colipase $(10 \mu \mathrm{g})$ et lipase pancréatique humaine $(5 \mu \mathrm{g})$. Un contrôle (courbe 5$)$ est réalisé avec ou sans injection de lipase gastrique humaine. Environ $15 \mu$ équivalents de soude sont, en moyenne, nécessaires pour amener le pH de 5,4 à 8,0.

Conditions expérimentales; $1 \mathrm{ml}$ d'une émulsion d'Intralipide à $30 \%$ dans $14 \mathrm{ml}$ de $\mathrm{NaCl}$, $150 \mathrm{mM} ; \mathrm{CaCl}_{2}, 6 \mathrm{mM}$; $\mathrm{BSA}, 30 \mathrm{mM}$; température $37^{\circ} \mathrm{C}$. 
al., 1986b). II est actuellement admis que la lipolyse intragastrique génère principalement des acides gras libres et des diglycérides (Hamosh et al., 1975, 1978). Nos observations montrent que, même pour un substrat finement émulsifié tel que I'Intralipide, la lipolyse intragastrique est essentielle pour permettre au niveau du duodénum I'hydrolyse ultérieure par la lipase pancréatique (fig. 6).

Borgström a observé que la phospholipase A2 d'origine pancréatique, utilisée dans un rapport molaire 35/1 par rapport à la lipase, hydrolyse les lécithines de l'émulsion d'Intralipide, donnant ainsi naissance à des acides gras libres qui favorisent la fixation du complexe lipase-colipase responsable de l'hydrolyse des triglycérides (Borgström, 1980).

Nos résultats montrent que la lipase pancréatique, associée à la phospholipase $A 2$, dans les proportions rencontrées dans le suc pancréatique humain, n'hydrolyse pas l'émulsion d'Intralipide (fig. 7, courbe 3). Par contre, une préincubation de cette émulsion avec du suc gastrique dans les conditions de $\mathrm{pH}$ existant dans l'estomac, permet ultérieurement au suc pancréatique humain d'hydrolyser rapidement les triglycérides. Dans cette dernière expérience, le rapport des activités lipasiques d'origine gastrique et pancréatique était de $2 / 1$.
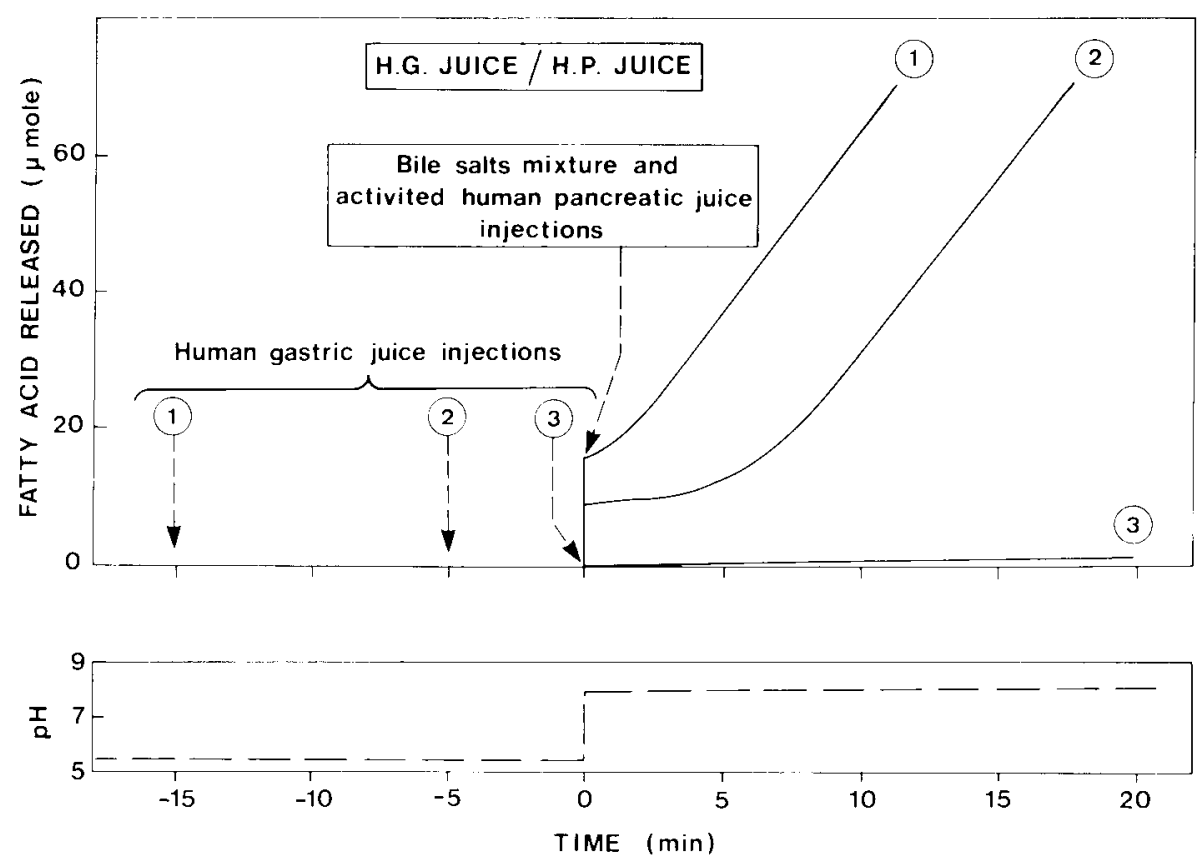

FIG. 7. - Cinétiques d'hydrolyse d'une émulsion d'intralipide par le suc pancréatique humain après différentes périodes d'incubation avec du suc gastrique humain.

Les flèches représentent les injections de suc gastrique à pH 5,4 (100 $\mu \mathrm{g}$ de protéines). Le pH est amené de 5,4 à 8,0 et l'on injecte simultanément un mélange de sels biliaires (concentration finale $8 \mathrm{mM}$ ) et du suc pancréatique humain activé $(50 \mu \mathrm{g}$ de protéines). Un contrôle est réalisé avec ou sans injection de suc gastrique. $15 \mu$ équivalents de soude sont, en moyenne, nécessaires pour amener le $\mathrm{pH}$ de 5,4 à 8,0 .

Conditions expérimentales : $1 \mathrm{ml}$ d'une émulsion d'Intralipide à $30 \%$ dans $14 \mathrm{ml}$ de $\mathrm{NaCl}$, $150 \mathrm{mM}$; $\mathrm{CaCl}^{2}, 6 \mathrm{mM}$; $\mathrm{BSA}, 30 \mu \mathrm{M}$; température $37^{\circ} \mathrm{C}$. 
Nos expériences réalisées in vitro, suggèrent fortement que l'action de la lipase gastrique humaine conditionne l'activité ultérieure de la lipase pancréatique, grâce aux acides gras libérés. In vivo, 10 à $30 \%$ des triglycérides alimentaires sont déjà hydrolysés au niveau gastrique (Hamosh et al., 1975, 1978). II semble donc que, dans ces conditions, la préhydrolyse gastrique génère un substrat directement hydrolysable dans l'intestin par la lipase pancréatique.

Nous avons observé (Gargouri et al., 1986b), en utilisant l'émulsion d'Intrali-

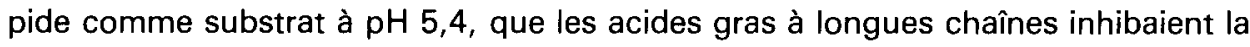
lipolyse catalysée par la lipase gastrique humaine. Une inhibition d'environ $80 \%$ est observée pour une concentration de $2 \mathrm{mM}$ en acides gras libres. Des expériences récentes montrent qu'une telle concentration en acides gras libres permet au contraire l'activation rapide (sans période de latence) du complexe lipase-colipase pancréatique à $\mathrm{pH} \mathrm{8,0} \mathrm{(fig.} \mathrm{7).} \mathrm{En} \mathrm{conclusion,} \mathrm{il} \mathrm{existerait} \mathrm{une} \mathrm{synergie} \mathrm{entre} \mathrm{les}$ actions des lipases de façon complémentaire à deux niveaux. L'hydrolyse enzymatique des lipides alimentaires au niveau gastrique est une étape importante de la digestion des lipides chez l'homme.

Journées Ingestion, Digestion, Absorption de l'Association française de Nutrition, Marseille, 5-6 décembre 1985.

Remerciements. - Les échantillons de suc gastrique humain sont dus à l'obligeance du Professeur H. Sarles (Laboratoire d'Hépatogastrœentérologie, Hôpital Sainte-Marguerite, 270, bd de Ste-Marguerite, 13009 Marseille). Nos remerciements vont également aux Drs A. De Caro et L. Multigner (INSERM U31, Traverse de la Gaye, 13009 Marseille) pour nous avoir fourni des échantillons de lipase pancréatique humaine pure.

\section{Références}

ABRAMS C. K., HAMOSH M., HUBBARD V. S., DUTTA S. K., HAMOSH P., 1984. Lingual lipase in cystic fibrosis. Quantitation of enzyme activity in the upper small intestine of patients with exocrine pancreatic insufficiency. J. clin. Invest., 73, 374-382.

BORGSTRÖM B., 1980. Importance of phospholipids, pancreatic phospholipase A2 and fatty acid for the digestion of dietary fat. Gastroenterology, 78, 954-962.

BORGSTRÖM B., DAHLQVIST A., LUNDTH G., SJOVALL J., 1957. Studies of intestinal digestion and absorption in the human. J. clin. Invest., 36, 1521-1528.

CAREY M. C., SMALL P. M., BLISS C. M., 1983. Lipid digestion and absorption. Ann. Rev. physiol., 45, 651-677.

CHAPUS C., SÉMÉRIVA M., 1976. Mechanism of pancreatic lipase action. 2. Catalytic properties of modified lipases. Biochemistry, 15, 4988-4991.

COHEN M., MORGAN G. R. H., HOFMAN A. F., 1971. Lipolytic activity of human gastric and duodenal juice against medium and long-chain triglycerides. Gastroenterology, 60, 1-15.

DE CARO J., BOUDOUARD M., BONICEL J., GUIDONI A., DESNUELLE P., ROVERY M., 1981. Porcine pancreatic lipase. Completion of the primary structure. Biochim. Biophys. Acta, 671, 129-138.

DOCHERTY A. J. P., BODMER M. W., ANGAL S., RIVIĖRE C., LOWE P. A., LYONS A., EMTAGE J. S., HARRIS T. J. R., 1985. Molecular cloning and nucleotide sequerice of rat lingual lipase cDNA. Nucleic Acid Res., 13, 1891-1903.

EASTWOOD M. A., HAMILTON D., 1970. Fatty acids in the lumen of the small intestine in man following a lipid-containing meal. Scand. J. Gastroenterol., 5, 225-230. 
FIELD R. B., SCOW R. O., 1983. Purification and characterization of rat lingual lipase. J. biol. Chem., 258, 14563-14569.

GARGOURI Y., JULIEN R., BOIS A. G., VERGER R., SARDA L., 1983. Studies on the detergent inhibition of pancreatic lipase activity. J. Lipid Res., 24, 1336-1342.

GARgOURI Y., JULIEN R., PIERONI G., VERGER R., SARDA L., 1984a. Studies on the inhibition of pancreatic and microbial lipases by soybean proteins. J. Lipid Res., 25, 1214-1221.

GARGOURI Y., JULIEN R., SUGIHARA A., VERGER R., SARDA L., 1984b. Inhibition of pancreatic and microbial lipases by proteins. Biochim. Biophys. Acta, 795, 326-331.

GARGOURI Y., PIERONI G., LOWE P. A., SARDA L., VERGER R., 1986a. Human gastric lipase. The effect of amphiphiles. Eur. J. Biochem., 156, 305-310.

GARGOURI Y., PIERONI G., SAUNIĖRE J.-F., LOWE P. A., SARDA L., VERGER R., 1986b. Imporance of human gastric lipase for duodenal lypolysis. An in vitro study. Biochim. Biophys. Acta (sous presse).

HAMOSH M., 1979. Fat digestion in the newborn: role of lingual lipase and preduodenal digestion. Pediatr. Res., 13, 615-622.

HAMOSH M., 1984. Lingual lipase, 49-81. In BORGSTRÖM B., BROCKMAN H. L., Lipases, Elsevier.

HAMOSH M., BURNS W. A., 1977. Lipolytic activity of human lingual glands (Ebner). Lab. Invest., 37, 603-308.

HAMOSH M., KLAEVEMAN H. L., WOLF R. D., SCOW R. O., 1975. Pharyngeal lipase and digestion of dietary triglyceride in man. J. clin. Invest., 55, 908-913.

HAMOSH M., SCANLON J. W., GANOT D., LIKEL M., SCANLON K. B., HAMOSH P., 1981. Fat digestion in the newborn : characterization of lipase in gastric aspirates of premature and term infants. J. clin. Invest., 67, 838-846.

HAMOSH M., SCOW R. O., 1973. Lingual lipase and its role in the digestion of dietary lipid. $J$. clin. Invest., 52, 88-95.

HAMOSH M., SIASUBRAMANIAN K. N., SALZMAN-MANN C., HAMOSH P., 1978. Fat digestion in the stomach cf premature infants. J. Pediatr., 93, 674-679.

HOFMANN A., BORGSTRÖM B., 1962. Physical-chemical state of lipids in intestinal content during their digestion and absorption. Fed. Proc., 21, 43-50.

JENSEN R. G., DE JONG F. A., CLARK R. M., PALMGREN L. G., LIAO T. H., HAMOSH M., 1982. Stereospecificity of premature human infant lingual lipase. Lipids, 17, 570-572.

JORPES E., MUTT V., 1966. Cholecystokinin and pancreozymin, one single hormone ? Acta physiol. Scand., 66, 196-202.

KLEIN E., LYMAN R. B., PETERSON L., 1967. The effect of lecithin on the activity of pancreatic lipase. Life Sci., 6, 1305-1307.

LAIRON D., NALBONE G., LAFONT H., LEONARDI J., DOMINGO N., HAUTON J. C., VERGER R., 1978. Possible roles of bile lipids and colipase in lipase adsorption. Biochemistry, 17, 52635269.

LINDSTRÖM M., LJUSBERG-WAHREN H., LARSSON K., 1981. Aqueous lipid phases of relevance to intestinal fat digestion and absorption. Lipids, 16, 749-754.

LINTHORST J. M., CLARK S. B., HOLT P. R., 1977. Triglyceride emulsification by amphipaths present in the intestinal lumen during digestion of fat. J. Colloid Interface Sci, 60, 1-10.

MUlLeR D. P. R., McCOlluM J. P. K., TROMPETER R. S., HARRIES J. T., 1975. Studies on the mechanism of fat absorption in congenital isolated lipase deficiency. Gut, 16, 838.

PATTON J. S., 1981. Gastrointestinal lipid digestion, 1123-1146. In JOHNSTON J. R., Physiology of the gastrointestinal tract, Raven Press, New York.

PATTON J. S., CAREY M. C., 1979. Watching fat digestion. Science, 204, 145-148.

PATTON J. S., CAREY M. C., 1981. Inhibition of human pancreatic lipase-colipase activity by mixed bile salt-phospholipid micelles. Am. J. Physiol., 241, G328-G336.

POPIELA T., SZAFRAN H., SZAFRAN Z., 1965. The activity of human gastric lipase towards p-nitrophenol esters. Clinica chim. Acta, 11, 283-285.

ROBERTS I. M., MONTGOMERY R. K., CAREY M. C., 1984. Rat lingual lipase : partial purification, hydrolytic properties, and comparison with pancreatic lipase. Am. Physiol. Soc., G385G393. 
SCHONHEYDER F., VOLQUARTZ K., 1946. The gastric lipase in man. Acta physiol. Scand., 11. 349-389.

SIURALA M., 1956. Lipase activity of the gastric mucosa of man under pathological conditions. Acta pathol. scand., 39, 268-278.

STERLING J. A., 1954. The common channel for bile and pancreatic ducts. Surg. Gynecol. Obstet., 98, 420-424.

SUGgS S. B., WALLACE R. B., HIROSE T., HAWASHIMA E. H., ITAKURA K., 1981. Proc. nat. Acad. Sci. USA, 78, 6613-6617.

TIRUPPATHI C., BALASUBRAMANIAN K. A., 1982. Purification and properties of an acid lipase from human gastric juice. Biochim. Biophys. Acta, 712, 692-697.

VERGER R., 1984. Pancreatic lipases, 121-150. In BORGSTRÖM B., BROCKMAN H. L., Lipases, Elsevier.

VOLHARD F., 1901. Uber das fettspaltende Ferment des Magens. Z. Klin. Med., 42, 414-429. 